Radial and Nonradial Pulsations as Probes of Stellar Physics

ASP Conference Series, Vol. 259, 2002

C. Aerts, T.R. Bedding, \& J. Christensen-Dalsgaard, eds.

\title{
Tidal Modulation of Radial Oscillations in Components of Circular-Orbit Close Binaries
}

\author{
T. Reyniers, P. Smeyers \\ Instituut voor Sterrenkunde, Katholieke Universiteit Leuven, Belgium
}

\begin{abstract}
A theoretical description is presented of the modulation of a radial oscillation in a uniformly rotating star in a close binary that is subject to the tidal action of a companion moving in a circular orbit. The star is assumed to rotate non-synchronously with the companion's orbital motion. A tidal modulation of a radial oscillation has been observed in the star $\theta$ Tucanae by Sterken (1997) and De Mey et al. (1998).
\end{abstract}

\section{Basic assumptions}

We consider a radially pulsating star in a close binary that is subject to the tidal action of a companion moving in a circular orbit. The star is assumed to rotate uniformly around an axis perpendicular to the orbital plane but nonsynchronously with the orbital motion of the companion, which is considered as a point mass. The star's time-dependent tidal distortion is taken into account to the first order in the small parameter $\varepsilon_{T}=\left(R_{1} / a\right)^{3}\left(M_{2} / M_{1}\right)$, where $R_{1}$ is the star's mean radius, $a$ the semi-major axis of the companion's relative orbit, $M_{1}$ the star's mass, and $M_{2}$ the companion's mass. The effects of the Coriolis force and the centrifugal force are neglected.

\section{Method}

The effect of the companion's tidal action on the star's radial pulsation is determined by means of an appropriate perturbation method. First, the equations that govern free, linear, isentropic oscillations in the tidally distorted star are transformed into equations defined in the domain of the unperturbed spherically symmetric star and, at each point in the star, the Lagrangian displacement is subject to a parallel displacement to the associated point in the same domain. Next, the resulting equations are solved by means of a two time variable expansion procedure (Kevorkian \& Cole, 1996).

To the first order in the parameter $\varepsilon_{T}$, the solution for the tidal perturbation of a radial oscillation mode $n$ in a spherically symmetric star, at a point with spherical coordinates $r, \theta, \phi$ at the instant $t$, takes the form 


$$
\begin{gathered}
(\delta r)_{n}(r, \theta, \phi ; t)=(\delta r)_{n, 0}(r)\left[A_{n, 0} \cos \left(\sigma_{n, 0} t\right)+B_{n, 0} \sin \left(\sigma_{n, 0} t\right)\right] \\
\left\{1+\varepsilon_{T}\left[\left(\sum_{\kappa \neq n} G_{\kappa}^{(1)}(r)\right) P_{2}(\cos \theta)\right.\right. \\
\left.\left.+2\left(\sum_{\lambda \neq n} G_{\lambda}^{(2)}(r)\right) P_{2}^{2}(\cos \theta) \cos 2[\phi+(\Omega-n) t]\right]\right\} .
\end{gathered}
$$

Here $A_{n, 0}$ and $B_{n, 0}$ are undetermined constants. The radial functions $G_{\kappa}^{(1)}(r)$ and $G_{\lambda}^{(2)}(r)$ are determined by the radial mode considered, the second-degree nonradial oscillation modes, and the tidal displacements generated by the companion. The first summation extends over the second-degree nonradial modes associated with the azimuthal number $m=0$, and the second summation over those associated with the azimuthal number $m=2$.

The tidal perturbation of a radial oscillation mode is of the order of $\varepsilon_{T}$, and consists of two parts. The first part is constant, the second part gives rise to a time-dependent modulation of the amplitude, which depends on the difference between the angular frequency of rotation $\Omega$ and the mean motion $n$.

\section{Application to the equilibrium model with uniform mass density}

In a first application, we have considered the tidal perturbation of radial oscillation modes of the equilibrium sphere with uniform mass density. In this case, we have restricted the summations to be over the contributions stemming from the $f$-mode, and the $p$ - and $g^{-}$-modes of the eight lowest radial orders. It follows that the constant part of the tidal perturbation is given by $\varepsilon_{T} 26.26 P_{2}(\cos \theta)$.

The time-dependent part of the tidal perturbation for the values 0.005 , 0.025 , and 0.05 of the ratio $(\Omega-n)^{2} /\left(G M_{1} / R_{1}^{3}\right)$ is given by

$$
\begin{array}{cc}
(\Omega-n)^{2} /\left(G M_{1} / R_{1}^{3}\right) & \text { Time-dependent part } \\
0.005 & -\varepsilon_{T} 5.54 P_{2}^{2}(\cos \theta) \cos [2(\phi+(\Omega-n) t] \\
0.025 & -\varepsilon_{T} 5.65 P_{2}^{2}(\cos \theta) \cos [2(\phi+(\Omega-n) t] \\
0.050 & -\varepsilon_{T} 5.95 P_{2}^{2}(\cos \theta) \cos [2(\phi+(\Omega-n) t] .
\end{array}
$$

One observes that the modulation of the amplitude increases with $(\Omega-n)$.

Application of the theory to polytropic models with increasing central mass condensations is in progress. It turns out that the contributions of a larger number of second-degree nonradial modes must be taken into account in the summations.

\section{References}

De Mey, K., Daems, K., \& Sterken, C. 1998, A\&A, 336, 527

Kevorkian, J. \& Cole, J.D. 1996, Multiple scale and perturbation methods (Springer Verlag)

Sterken, C. 1997, A\&A, 325, 563 